CRYSTALLOGRAPHIC COMMUNICATIONS

ISSN 2056-9890

Received 13 May 2021

Accepted 1 June 2021

Edited by M. Weil, Vienna University of Technology, Austria

Keywords: crystal structure; pyridine; styryl; ester; hydrogen bond; Hirshfeld surface analysis.

CCDC reference: 2087180

Supporting information: this article has supporting information at journals.iucr.org/e
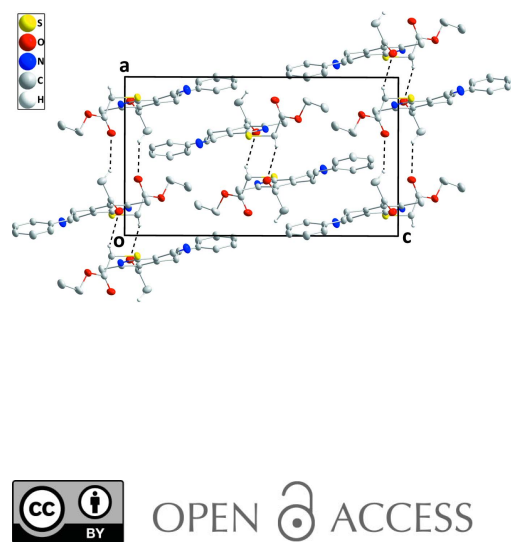

\section{Crystal structure and Hirshfeld surface analysis of ethyl 2-(\{5-acetyl-3-cyano-6-methyl-4-[(E)-2- phenylethenyl]pyridin-2-yl\}sulfanyl)acetate}

\author{
Safiyyah A. H. Al-Waleedy, ${ }^{a *}$ Shaaban K. Mohamed, ${ }^{b, c} *$ Joel T. Mague, ${ }^{d}$ Mehmet \\ Akkurt, $^{e}$ Mohamed S. Abbady ${ }^{f}$ and Etify A. Bakhite ${ }^{f}$
}

\begin{abstract}
${ }^{a}$ Department of Chemistry, Faculty of Science, Taiz University, Taiz, Yemen, ${ }^{\mathbf{b} C h e m i s t r y}$ and Environmental Division, Manchester Metropolitan University, Manchester, M1 5GD, England, ' ${ }^{\mathbf{C}}$ Chemistry Department, Faculty of Science, Minia University, 61519 El-Minia, Egypt, 'Department of Chemistry, Tulane University, New Orleans, LA 70118, USA, ${ }^{\mathbf{e}}$ Department of Physics, Faculty of Sciences, Erciyes University, 38039 Kayseri, Turkey, and ${ }^{\mathbf{f} C h e m i s t r y ~ D e p a r t m e n t, ~}$ Faculty of Science, Assiut University, Assiut 71516, Egypt. *Correspondence e-mail: safiyyahalwaleedy@gmail.com, shaabankamel@yahoo.com
\end{abstract}

In the title molecule, $\mathrm{C}_{21} \mathrm{H}_{20} \mathrm{~N}_{2} \mathrm{O}_{3} \mathrm{~S}$, the styryl and ester substituents are displaced to opposite sides of the plane of the pyridine ring. In the crystal, $\mathrm{C}-$ $\mathrm{H} \cdots \mathrm{O}$ hydrogen bonds form chains extending parallel to the $a$-axis direction, which pack with partial intercalation of the styryl and ester substituents. A Hirshfeld surface analysis indicates that the most significant contributions to the crystal packing are from $\mathrm{H} \cdots \mathrm{H}(43.6 \%), \mathrm{C} \cdots \mathrm{H} / \mathrm{H} \cdots \mathrm{C}(15.6 \%), \mathrm{O} \cdots \mathrm{H} / \mathrm{H} \cdots \mathrm{O}$ $(14.9 \%)$ and $\mathrm{N} \cdots \mathrm{H} / \mathrm{H} \cdots \mathrm{N}(11.2 \%)$ contacts.

\section{Chemical context}

Numerous pyridine-containing natural products and synthetic organic compounds with various biophysio- and pharmacological activities have been reported (Gibson et al., 2007; Vidaillac et al., 2007). These scaffolds are also of widespread interest in supramolecular and coordination chemistry, as well as for materials science (Balasubramanian \& Keay, 1996). The above findings promoted us to study the crystal structure of the title compound, $\mathrm{C}_{21} \mathrm{H}_{20} \mathrm{~N}_{2} \mathrm{O}_{3} \mathrm{~S}$.<smiles>CCOC(=O)CSc1nc(C)c(C(C)=O)c(/C=C/c2ccccc2)c1C#N</smiles>

\section{Structural commentary}

The styryl substituent and the ester group are displaced to opposite sides of the plane of the pyridine ring (Fig. 1). The dihedral angle between the mean planes of the phenyl (C8$\mathrm{C} 13)$ and pyridine (N1/C1-C5) rings is $27.86(3)^{\circ}$. The $\mathrm{C} 1-$ $\mathrm{C} 2-\mathrm{C} 14-\mathrm{C} 15$ torsion angle of $68.1(2)^{\circ}$ indicates that the 


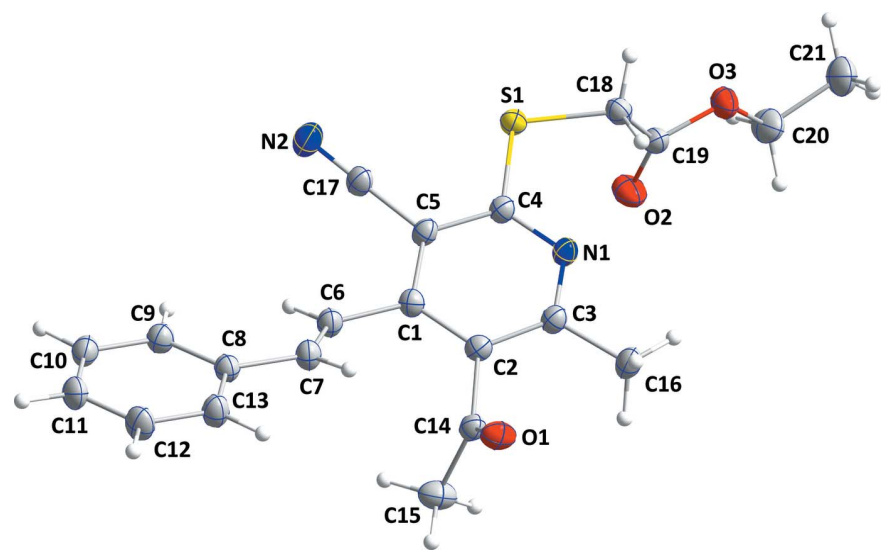

Figure 1

The title molecule with labelling scheme and displacement ellipsoids at the $50 \%$ probability level.

acetyl group is rotated well out of the plane of the pyridine ring, while the $\mathrm{N} 1-\mathrm{C} 4-\mathrm{S} 1-\mathrm{C} 18$ torsion angle of $-5.66(12)^{\circ}$ shows that the link to the ester group is nearly coplanar with the pyridine ring.

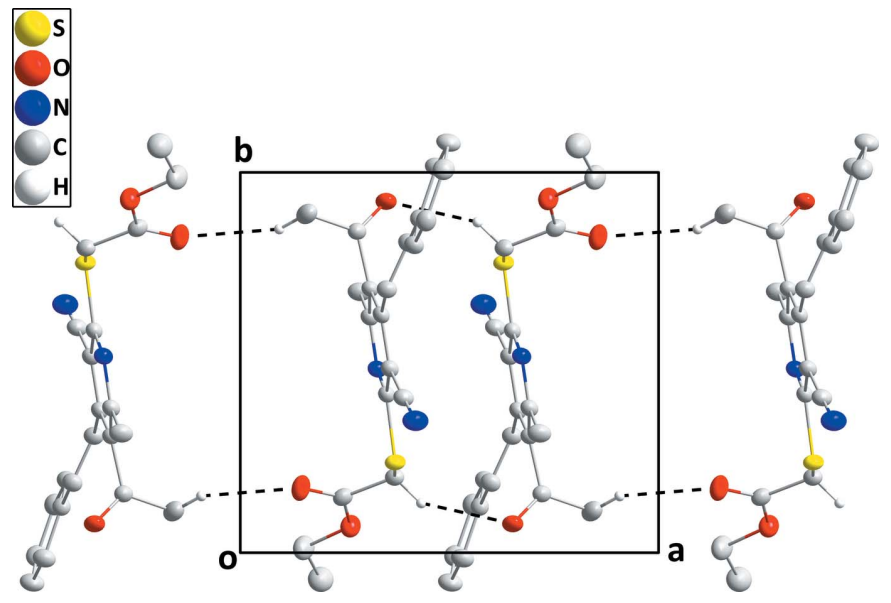

Figure 2

A portion of one hydrogen-bonded chain in a view along the $c$-axis direction. $\mathrm{C}-\mathrm{H} \cdots \mathrm{O}$ hydrogen bonds are depicted by dashed lines.

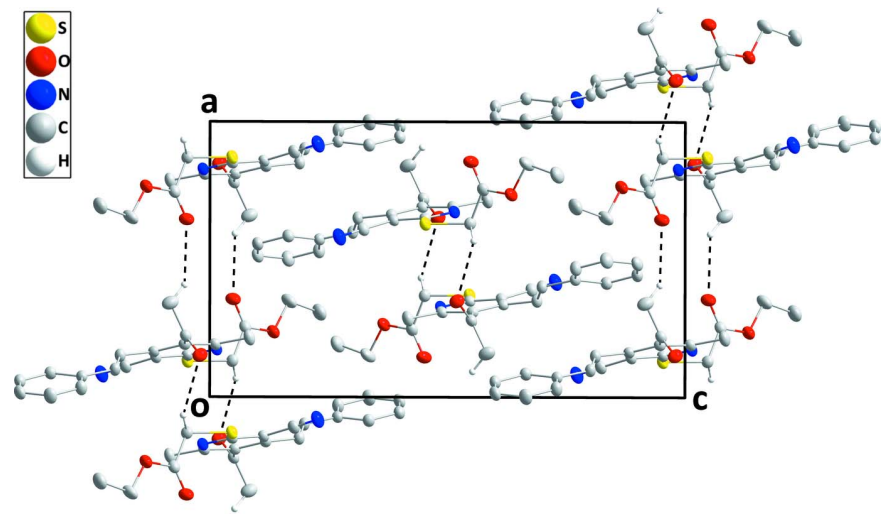

Figure 3

Packing of the molecules in the title compound in a view along the $b$-axis direction. $\mathrm{C}-\mathrm{H} \cdots \mathrm{O}$ hydrogen bonds are depicted by dashed lines.
Table 1

Hydrogen-bond geometry $\left(\AA{ }^{\circ}{ }^{\circ}\right)$.

\begin{tabular}{lllll}
\hline$D-\mathrm{H} \cdots A$ & $D-\mathrm{H}$ & $\mathrm{H} \cdots A$ & $D \cdots A$ & $D-\mathrm{H} \cdots A$ \\
\hline $\mathrm{C} 15-\mathrm{H} 15 A \cdots \mathrm{O} 2^{\mathrm{i}}$ & $0.98(2)$ & $2.56(2)$ & $3.375(2)$ & $139.9(17)$ \\
$\mathrm{C} 18-\mathrm{H} 18 B \cdots \mathrm{O} 1^{\mathrm{ii}}$ & $0.965(17)$ & $2.493(17)$ & $3.2989(17)$ & $140.9(13)$ \\
\hline
\end{tabular}

Symmetry codes: (i) $-x+2,-y+1,-z+1$; (ii) $-x+1,-y+1,-z+1$.

Table 2

Summary of short interatomic contacts $(\AA)$ in the title compound.

\begin{tabular}{lll}
\hline Contact & Distance & Symmetry operation \\
\hline $\mathrm{H} 20 B \cdots \mathrm{H} 16 B$ & 2.53 & $x, 1+y, z$ \\
$\mathrm{H} 18 B \cdots \mathrm{H} 7$ & 2.42 & $1-x, 1-y, 1-z$ \\
$\mathrm{O} 2 \cdots \mathrm{H} 10$ & 2.613 & $\frac{3}{2}-x, \frac{1}{2}+y, \frac{1}{2}-z$ \\
$\mathrm{H} 15 A \cdots \mathrm{O} 2$ & 2.56 & $2-x, 1-y, 1-z$ \\
$\mathrm{~N} 2 \cdots \mathrm{H} 20 A$ & 2.63 & $-\frac{1}{2}+x, \frac{3}{2}-y,-\frac{1}{2}+z$ \\
$\mathrm{H} 11 \cdots \mathrm{H} 11$ & 2.31 & $1-x,-y,-z$ \\
$\mathrm{H} 12 \cdots \mathrm{H} 20 A$ & 2.47 & $-\frac{1}{2}+x, \frac{1}{2}-y,-\frac{1}{2}+z$ \\
$\mathrm{H} 16 A \cdots \mathrm{H} 21 B$ & 2.49 & $\frac{3}{2}-x,-\frac{1}{2}+y, \frac{3}{2}-z$ \\
\hline
\end{tabular}

\section{Supramolecular features}

In the crystal, inversion dimers are formed by intermolecular $\mathrm{C} 15-\mathrm{H} 15 A \cdots \mathrm{O} 2$ hydrogen bonds between a methyl $\mathrm{H}$ atom of the acetyl group and the carbonyl $\mathrm{O}$ atom of the ester function. These dimers are further linked by inversion-related $\mathrm{C} 18-\mathrm{H} 18 \mathrm{~B} \ldots \mathrm{O} 1 \mathrm{hydrogen}$ bonds between a methylene $\mathrm{H}$ atom and the carbonyl $\mathrm{O}$ atom of the acetyl group (Table 1) to form ribbons of molecules extending parallel to the $a$-axis direction (Fig. 2). The chains pack with a partial intercalation of the styryl and ester substituents (Fig. 3).

\section{Hirshfeld surface analysis}

To quantify the intermolecular interactions in the title compound, a Hirshfeld surface analysis was performed and two-dimensional fingerprint plots were generated using Crystal Explorer (Turner et al., 2017). The Hirshfeld surface mapped over $d_{\text {norm }}$ in the range -0.1607 to +1.3888 arbitrary

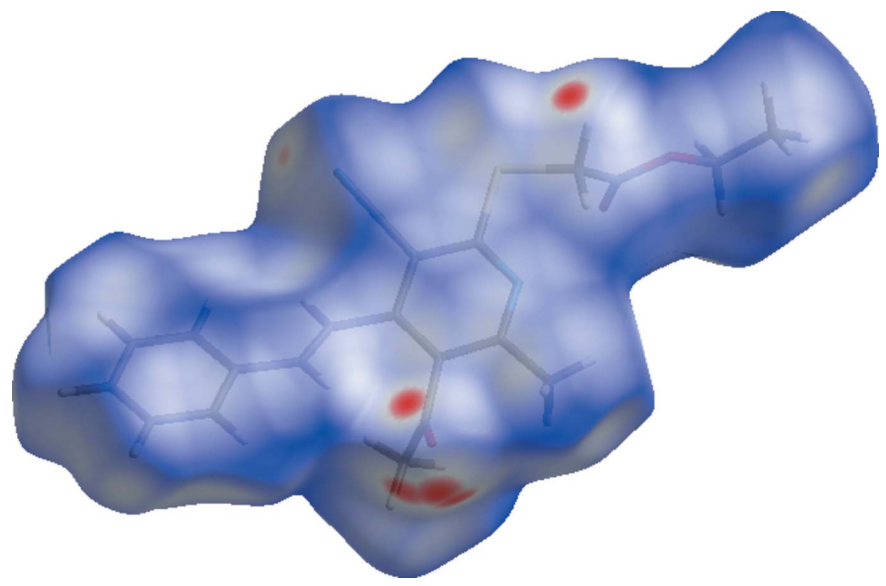

Figure 4

A view of the three-dimensional Hirshfeld surface for the title compound, plotted over $d_{\text {norm }}$ in the range -0.1607 to +1.3888 a.u. 
units is depicted in Fig. 4, where the red regions indicate apparent hydrogen bonds in this structure. The intensities of the red areas are greater for $\mathrm{C} 15-\mathrm{H} 15 A \cdots \mathrm{O} 2$ and $\mathrm{C} 18-$ $\mathrm{H} 18 B \cdots \mathrm{O} 1$, indicating the strongest interactions as compared to other red spots on the Hirshfeld surface; Table 2 lists corresponding close intermolecular contacts. The two-dimensional fingerprint plots (Fig. 5) reveal that the largest contributions are from $\mathrm{H} \cdots \mathrm{H}(43.6 \%$; Fig. $5 b), \mathrm{C} \cdots \mathrm{H} / \mathrm{H} \cdots \mathrm{C}$

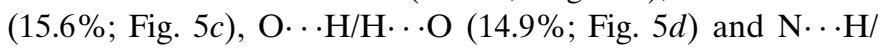
$\mathrm{H} \cdots \mathrm{N}(11.2 \%$; Fig. 5e) interactions. Other interactions contributing less to the crystal packing are $\mathrm{S} \cdots \mathrm{H} / \mathrm{H} \cdots \mathrm{S}$ $(5.9 \%), \mathrm{C} \cdots \mathrm{C}(4.4 \%), \mathrm{N} \cdots \mathrm{C} / \mathrm{C} \cdots \mathrm{N}(1.5 \%), \mathrm{S} \cdots \mathrm{O} / \mathrm{O} \cdots \mathrm{S}$ $(1.1 \%), \mathrm{O} \cdots \mathrm{C} / \mathrm{C} \cdots \mathrm{O}(1.0 \%), \mathrm{O} \cdots \mathrm{O}(0.3 \%), \mathrm{N} \cdots \mathrm{N}(0.2 \%)$ and $\mathrm{S} \cdots \mathrm{C} / \mathrm{C} \cdots \mathrm{S}(0.2 \%)$.

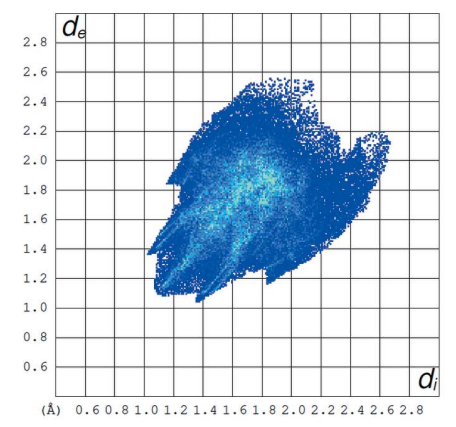

(a) All...All

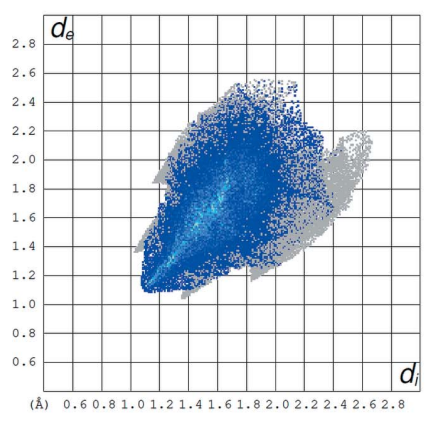

(b) $H$...H

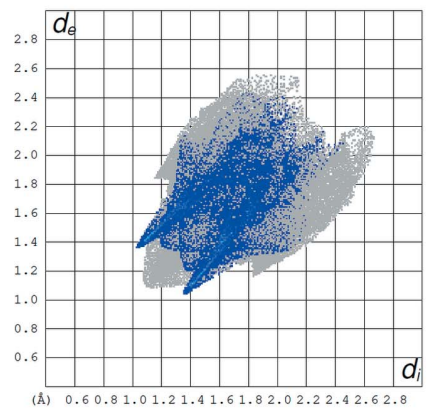

(d) $\mathrm{O} \ldots \mathrm{H} / \mathrm{H} \ldots \mathrm{O}$

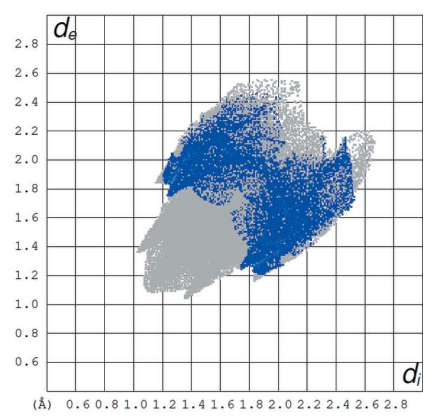

(c) $\mathrm{C} \ldots \mathrm{H} / \mathrm{H} \ldots \mathrm{C}$

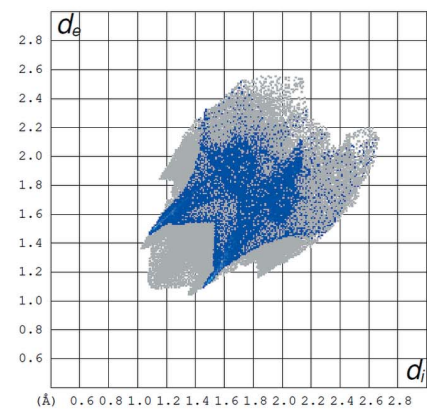

(e) $N \ldots H / H \ldots N$
Figure 5

A view of the two-dimensional fingerprint plots for the title compound, showing $(a)$ all interactions, and delineated into $(b) \mathrm{H} \cdots \mathrm{H},(c) \mathrm{C} \cdots \mathrm{H} /$ $\mathrm{H} \cdots \mathrm{C},(d) \mathrm{O} \cdots \mathrm{H} / \mathrm{H} \cdots \mathrm{O}$ and $(e) \mathrm{N} \cdots \mathrm{H} / \mathrm{H} \cdots \mathrm{N}$ interactions. The $d_{\mathrm{i}}$ and $d_{\mathrm{e}}$ values are the closest internal and external distances (in $\AA$ ) from given points on the Hirshfeld surface.

\section{Database survey}

A search of the Cambridge Structural Database (version 5.42, update 1, Feb 2021; Groom et al., 2016) for related structures with the 2-sulfanylpyridine-3-carbonitrile moiety of the title compound gave the following matches: ethyl 4-methyl-2phenyl-6-thioxo-1,6-dihydro-5-pyrimidinecarboxylate monohydrate (DEWCIS; Cunha et al., 2007), ethyl 4-(5-ethoxycarbonyl-6-methyl-2-phenyl-4-pyrimidinyldisulfanyl)-6-methyl-2-phenyl-5-pyrimidinecarboxylate (DEWCAK; Cunha et al., 2007), ethyl 4-\{[(4-chlorophenyl)methyl]sulfanyl\}-6-methyl-2-phenylpyrimidine-5-carboxylate (NILKOL; Stolarczyk et al., 2018), (4-\{[(4-chlorophenyl)methyl]sulfanyl\}-6-methyl-2phenylpyrimidin-5-yl)methanol (NILKUR; Stolarczyk et al., 2018) and 4-\{[(4-chlorophenyl)methyl]sulfanyl\}-5,6-dimethyl2-phenylpyrimidine (NILLAY; Stolarczyk et al., 2018).

Compound DEWCIS crystallizes in the space group $P 2_{1} / c$ with one molecule in the asymmetric unit. $\mathrm{N}-\mathrm{H} \cdots \mathrm{O}, \mathrm{O}-$ $\mathrm{H} \cdots \mathrm{N}$ and $\mathrm{O}-\mathrm{H} \cdots \mathrm{S}$ interactions involving the water molecules, as well as $\pi-\pi$ stacking interactions between the molecules along the $b$ axis contribute to the formation of layers parallel to the $b c$ plane. The stability of the molecular packing is achieved by van der Waals interactions between these layers. Compound DEWCAK crystallizes in the space group $P \overline{1}$ with one molecule in the asymmetric unit. In the crystal structure of DEWCAK, there are no classical hydrogen bonds. The molecular packing is stabilized by $\mathrm{C}-\mathrm{H} \cdots \pi$ interactions and $\pi-\pi$ stacking interactions. Compound NILKOL crystallizes in the space group $P \overline{1}$ with one molecule in the asymmetric unit, whereas compounds NILKUR and NILLAY crystallize in the space group $P 2_{1} / c$ with two and one molecules, respectively, in their asymmetric units. The conformation of each molecule is best defined by the dihedral angles formed between the pyrimidine ring and the planes of the two aryl substituents attached at the 2- and 4-positions. The only structural difference between the three compounds is the substituent at the 5-position of the pyrimidine ring, but they present significantly different features in their hydrogenbonding interactions. NILKOL displays a chain structure whereby the chains are further extended into a two-dimensional network. In NILKUR and NILLAY, the hydrogenbonded chains have no further aggregation.

\section{Synthesis and crystallization}

A mixture of 5-acetyl-3-cyano-6-methyl-4-styrylpyridine$2(1 \mathrm{H})$-thione $(3.24 \mathrm{~g}, 10 \mathrm{mmol})$, ethyl chloroacetate $(1.1 \mathrm{ml}$, $10 \mathrm{mmol})$ and sodium acetate trihydrate $(1.5 \mathrm{~g}, 11 \mathrm{mmol})$ in ethanol $(40 \mathrm{ml})$ was heated under reflux for $30 \mathrm{~min}$. The solid that formed after dilution with water $(20 \mathrm{ml})$ was filtered off and recrystallized from methanol in the form of fine colourless crystals of the title compound, yield 85\%; m.p. 343-344 K. Its IR spectrum showed characteristic absorption bands at $2219 \mathrm{~cm}^{-1}$ for $(\mathrm{C} \equiv \mathrm{N})$, at $1748 \mathrm{~cm}^{-1}$ for $(\mathrm{C}=\mathrm{O}$, non conjugated ester) and at $1724 \mathrm{~cm}^{-1}$ for $(\mathrm{C}=\mathrm{O}$, conjugated ester $)$. The ${ }^{1} \mathrm{H}$ NMR spectrum $\left(400 \mathrm{MHz}\right.$, DMSO- $d_{6}$ ) displayed a multiplet at $\delta=6.60-7.63 \mathrm{ppm}(7 \mathrm{H}: \mathrm{CH}=\mathrm{CH}$ and $\mathrm{Ar}-\mathrm{Hs})$, a 
multiplet at $\delta=4.16-4.37 \mathrm{ppm}\left(6 \mathrm{H}\right.$ : two $\mathrm{OCH}_{2}$ and $\left.\mathrm{SCH}_{2}\right)$, a singlet at $\delta=2.52 \mathrm{ppm}\left(3 \mathrm{H}, \mathrm{CH}_{3}\right.$ at $\mathrm{C}-6$, overlapped with solvent signal) and a multiplet at $\delta=1.21-1.27 \mathrm{ppm}(6 \mathrm{H}$ : two $\mathrm{CH}_{3}$ of ester groups).

\section{Refinement}

Crystal data, data collection and structure refinement details are summarized in Table 3. The $\mathrm{C}$-bound $\mathrm{H}$ atoms were refined freely, while the $\mathrm{H}$ atoms of the $\mathrm{C} 16$ methyl group were placed geometrically $(\mathrm{C}-\mathrm{H}=0.98 \AA$ ) and refined as riding atoms with $U_{\text {iso }}(\mathrm{H})=1.5 U_{\text {eq }}(\mathrm{C})$.

\section{Acknowledgements}

Author contributions are as follows: Conceptualization, EAB, MSA and SKM; methodology, JTM, EAB and MA; investigation, JTM, SKM, and EAB; writing (original draft), JTM, AM, SKM and EAB; writing (review and editing of the manuscript), MA and SKM; visualization, MA, SKM and JTM; funding acquisition, SAHA and SKM; resources, MA, JTM, EAB and SKM. AAA, VNK and FNN; supervision, SKM and MA.

\section{Funding information}

The support of NSF-MRI grant No. 1228232 for the purchase of the diffractometer and Tulane University for support of the Tulane Crystallography Laboratory are gratefully acknowledged.

\section{References}

Balasubramanian, M. \& Keay, J. G. (1996). In Comprehensive Heterocyclic Chemistry II, edited by A. R Katritzky, C. W. Rees \& E. F. V. Scriven, pp. 245-300. Oxford: Pergamon.

Brandenburg, K. \& Putz, H. (2012). DIAMOND, Crystal Impact GbR, Bonn, Germany.

Bruker (2016). APEX3 and SAINT. Bruker AXS, Inc., Madison, Wisconsin, USA.

Cunha, S., Bastos, R. M., Silva, P. O., Nobre Costa, G. A., Vencato, I., Lariucci, C., Napolitano, H. B., de Oliveira, C. M. A., Kato, L., da Silva, C. C., Menezes, D. \& Vannier-Santos, M. A. (2007). Monatsh. Chem. 138, 111-119.

Gibson, V. C., Redshaw, C. \& Solan, G. A. (2007). Chem. Rev. 107, 1745-1776.

Groom, C. R., Bruno, I. J., Lightfoot, M. P. \& Ward, S. C. (2016). Acta Cryst. B72, 171-179.
Table 3

Experimental details.

\begin{tabular}{|c|c|}
\hline \multicolumn{2}{|l|}{ Crystal data } \\
\hline Chemical formula & $\mathrm{C}_{21} \mathrm{H}_{20} \mathrm{~N}_{2} \mathrm{O}_{3} \mathrm{~S}$ \\
\hline$M_{\mathrm{r}}$ & 380.45 \\
\hline Crystal system, space group & Monoclinic, $P 2_{1} / n$ \\
\hline Temperature $(\mathrm{K})$ & 150 \\
\hline$a, b, c(\AA)$ & $10.7365(4), 9.7590(3), 18.5600(7)$ \\
\hline$\beta\left(^{\circ}\right)$ & $90.066(1)$ \\
\hline$V\left(\AA^{3}\right)$ & $1944.67(12)$ \\
\hline$Z$ & 4 \\
\hline Radiation type & $\mathrm{Cu} K \alpha$ \\
\hline$\mu\left(\mathrm{mm}^{-1}\right)$ & 1.67 \\
\hline Crystal size $(\mathrm{mm})$ & $0.27 \times 0.12 \times 0.05$ \\
\hline \multicolumn{2}{|l|}{ Data collection } \\
\hline Diffractometer & $\begin{array}{l}\text { Bruker D8 VENTURE PHOTON } \\
100 \text { CMOS }\end{array}$ \\
\hline Absorption correction & $\begin{array}{l}\text { Multi-scan (SADABS; Krause et } \\
\quad \text { al., 2015) }\end{array}$ \\
\hline$T_{\min }, T_{\max }$ & $0.80,0.92$ \\
\hline $\begin{array}{l}\text { No. of measured, independent and } \\
\text { observed }[I>2 \sigma(I)] \text { reflections }\end{array}$ & $14722,3912,3520$ \\
\hline$R_{\text {int }}$ & 0.034 \\
\hline$(\sin \theta / \lambda)_{\max }\left(\AA^{-1}\right)$ & 0.625 \\
\hline \multicolumn{2}{|l|}{ Refinement } \\
\hline$R\left[F^{2}>2 \sigma\left(F^{2}\right)\right], w R\left(F^{2}\right), S$ & $0.033,0.088,1.04$ \\
\hline No. of reflections & 3912 \\
\hline No. of parameters & 314 \\
\hline $\mathrm{H}$-atom treatment & $\begin{array}{l}\mathrm{H} \text { atoms treated by a mixture of } \\
\text { independent and constrained } \\
\text { refinement }\end{array}$ \\
\hline$\Delta \rho_{\max }, \Delta \rho_{\min }\left(\mathrm{e} \AA^{-3}\right)$ & $0.22,-0.29$ \\
\hline
\end{tabular}

Computer programs: APEX3 and SAINT (Bruker, 2016), SHELXT (Sheldrick, 2015a), SHELXL (Sheldrick, 2015b), DIAMOND (Brandenburg \& Putz, 2012) and publCIF (Westrip, 2010).

Krause, L., Herbst-Irmer, R., Sheldrick, G. M. \& Stalke, D. (2015). J. Appl. Cryst. 48, 3-10.

Sheldrick, G. M. (2015a). Acta Cryst. A71, 3-8.

Sheldrick, G. M. (2015b). Acta Cryst. C71, 3-8.

Stolarczyk, M., Bryndal, I., Matera-Witkiewicz, A., Lis, T., Królewska-Golińska, K., Cieślak, M., Kaźmierczak-Barańska, J. \& Cieplik, J. (2018). Acta Cryst. C74, 1138-1145.

Turner, M. J., McKinnon, J. J., Wolff, S. K., Grimwood, D. J., Spackman, P. R., Jayatilaka, D. \& Spackman, M. A. (2017). Crystal Explorer 17. University of Western Australia. http://hirshfeldsurface.net

Vidaillac, C., Guillon, J., Arpin, C., Forfar-Bares, I., Ba, B. B., Grellet, J., Moreau, S., Caignard, D. H., Jarry, C. \& Quentin, C. (2007). Antimicrob. Agents Chemother. 51, 831-838.

Westrip, S. P. (2010). J. Appl. Cryst. 43, 920-925. 


\section{supporting information}

Acta Cryst. (2021). E77, 730-733 [https://doi.org/10.1107/S2056989021005600]

\section{Crystal structure and Hirshfeld surface analysis of ethyl 2-(\{5-acetyl-3-cyano-6- methyl-4-[(E)-2-phenylethenyl]pyridin-2-yl\}sulfanyl)acetate}

\section{Safiyyah A. H. Al-Waleedy, Shaaban K. Mohamed, Joel T. Mague, Mehmet Akkurt, Mohamed}

\section{S. Abbady and Etify A. Bakhite}

\section{Computing details}

Data collection: APEX3 (Bruker, 2016); cell refinement: SAINT (Bruker, 2016); data reduction: SAINT (Bruker, 2016); program(s) used to solve structure: SHELXT (Sheldrick, 2015a); program(s) used to refine structure: SHELXL (Sheldrick, 2015b); molecular graphics: DIAMOND (Brandenburg \& Putz, 2012); software used to prepare material for publication: publCIF (Westrip, 2010).

Ethyl 2-(\{5-acetyl-3-cyano-6-methyl-4-[(E)-2-phenylethenyl]pyridin-2-yl\}sulfanyl)acetate

\section{Crystal data}

$\mathrm{C}_{21} \mathrm{H}_{20} \mathrm{~N}_{2} \mathrm{O}_{3} \mathrm{~S}$

$M_{r}=380.45$

Monoclinic, $P 2{ }_{1} / n$

$a=10.7365$ (4) $\AA$

$b=9.7590(3) \AA$

$c=18.5600(7) \AA$

$\beta=90.066(1)^{\circ}$

$V=1944.67(12) \AA^{3}$

$Z=4$

\section{Data collection}

Bruker D8 VENTURE PHOTON 100 CMOS diffractometer

Radiation source: INCOATEC I $\mu \mathrm{S}$ micro-focus source

Mirror monochromator

Detector resolution: 10.4167 pixels $\mathrm{mm}^{-1}$

$\omega$ scans

Absorption correction: multi-scan

(SADABS; Krause et al., 2015)

\section{Refinement}

Refinement on $F^{2}$

Least-squares matrix: full

$R\left[F^{2}>2 \sigma\left(F^{2}\right)\right]=0.033$

$w R\left(F^{2}\right)=0.088$

$S=1.04$

3912 reflections

314 parameters

0 restraints
$F(000)=800$

$D_{\mathrm{x}}=1.299 \mathrm{Mg} \mathrm{m}^{-3}$

$\mathrm{Cu} K \alpha$ radiation, $\lambda=1.54178 \AA$

Cell parameters from 9951 reflections

$\theta=4.8-74.6^{\circ}$

$\mu=1.67 \mathrm{~mm}^{-1}$

$T=150 \mathrm{~K}$

Column, colourless

$0.27 \times 0.12 \times 0.05 \mathrm{~mm}$

$T_{\min }=0.80, T_{\max }=0.92$

14722 measured reflections

3912 independent reflections

3520 reflections with $I>2 \sigma(I)$

$R_{\text {int }}=0.034$

$\theta_{\text {max }}=74.6^{\circ}, \theta_{\min }=4.8^{\circ}$

$h=-13 \rightarrow 12$

$k=-11 \rightarrow 12$

$l=-21 \rightarrow 22$

Primary atom site location: dual

Secondary atom site location: difference Fourier map

Hydrogen site location: mixed

$\mathrm{H}$ atoms treated by a mixture of independent and constrained refinement

$w=1 /\left[\sigma^{2}\left(F_{\mathrm{o}}^{2}\right)+(0.0426 P)^{2}+0.7692 P\right]$ where $P=\left(F_{\mathrm{o}}^{2}+2 F_{\mathrm{c}}^{2}\right) / 3$ 
$(\Delta / \sigma)_{\max }=0.001$

$\Delta \rho_{\max }=0.22$ e $\AA^{-3}$

$\Delta \rho_{\min }=-0.28$ e $\AA^{-3}$
Extinction correction: SHELXL 2018/3

(Sheldrick, 2015b),

$\mathrm{Fc}^{*}=\mathrm{kFc}\left[1+0.001 \times \mathrm{Fc}^{2} \lambda^{3} / \sin (2 \theta)\right]^{-1 / 4}$

Extinction coefficient: $0.0041(3)$

\section{Special details}

Geometry. All esds (except the esd in the dihedral angle between two 1.s. planes) are estimated using the full covariance matrix. The cell esds are taken into account individually in the estimation of esds in distances, angles and torsion angles; correlations between esds in cell parameters are only used when they are defined by crystal symmetry. An approximate (isotropic) treatment of cell esds is used for estimating esds involving l.s. planes.

Refinement. Refinement of $\mathrm{F}^{2}$ against ALL reflections. The weighted R-factor $\mathrm{wR}$ and goodness of fit $\mathrm{S}$ are based on $\mathrm{F}^{2}$, conventional R-factors $R$ are based on $F$, with $F$ set to zero for negative $F^{2}$. The threshold expression of $F^{2}>2 \operatorname{sigma}\left(\mathrm{F}^{2}\right)$ is used only for calculating R-factors(gt) etc. and is not relevant to the choice of reflections for refinement. R-factors based on $\mathrm{F}^{2}$ are statistically about twice as large as those based on $\mathrm{F}$, and R- factors based on ALL data will be even larger. Independent refinement of the hydrogen atoms attached to C16 led to an unreasonable geometry so these were included as riding contributions $(\mathrm{C}-\mathrm{H}=0.98 \AA)$ with an AFIX 137 instruction.

Fractional atomic coordinates and isotropic or equivalent isotropic displacement parameters $\left(\AA^{2}\right)$

\begin{tabular}{|c|c|c|c|c|}
\hline & $x$ & $y$ & $z$ & $U_{\text {iso }} * / U_{\text {eq }}$ \\
\hline $\mathrm{S} 1$ & $0.62964(3)$ & 0.76240 & $0.45327(2)$ & $0.02319(11)$ \\
\hline $\mathrm{O} 1$ & $0.65133(9)$ & $0.07606(9)$ & $0.47995(6)$ & $0.0295(2)$ \\
\hline $\mathrm{O} 2$ & $0.85513(10)$ & $0.82982(12)$ & $0.54910(6)$ & $0.0367(3)$ \\
\hline $\mathrm{O} 3$ & $0.73641(9)$ & $0.93032(11)$ & $0.63373(5)$ & $0.0304(2)$ \\
\hline N1 & $0.67271(10)$ & $0.51703(11)$ & $0.51422(6)$ & $0.0222(2)$ \\
\hline N2 & $0.58138(13)$ & $0.65298(13)$ & $0.27261(7)$ & 0.0351 \\
\hline $\mathrm{C} 1$ & $0.66330(11)$ & $0.37425(13)$ & $0.38230(7)$ & 0.0203 \\
\hline $\mathrm{C} 2$ & $0.69019(12)$ & $0.30604(13)$ & $0.44698(7)$ & 0.0202 \\
\hline $\mathrm{C} 3$ & $0.69103(12)$ & $0.38044(13)$ & $0.51167(7)$ & 0.0215 \\
\hline $\mathrm{C} 4$ & $0.65042(12)$ & $0.58371(13)$ & $0.45305(7)$ & $0.0201(3)$ \\
\hline $\mathrm{C} 5$ & $0.64281(12)$ & $0.51610(13)$ & $0.38647(7)$ & 0.0208 \\
\hline C6 & $0.64972(13)$ & $0.30725(14)$ & $0.31165(7)$ & 0.0233 \\
\hline H6 & $0.6751(17)$ & $0.363(2)$ & $0.2711(10)$ & $0.038(5)^{*}$ \\
\hline $\mathrm{C} 7$ & $0.59846(13)$ & $0.18469(14)$ & $0.30086(7)$ & $0.0239(3)$ \\
\hline $\mathrm{H} 7$ & $0.5717(16)$ & $0.1327(18)$ & $0.3420(10)$ & $0.033(4)^{*}$ \\
\hline $\mathrm{C} 8$ & $0.57238(12)$ & $0.12114(13)$ & $0.23083(7)$ & $0.0222(3)$ \\
\hline $\mathrm{C} 9$ & $0.58480(13)$ & $0.19075(14)$ & $0.16518(7)$ & 0.0245 \\
\hline H9 & $0.6136(17)$ & $0.284(2)$ & $0.1648(9)$ & $0.034(5)^{*}$ \\
\hline $\mathrm{C} 10$ & $0.55575(13)$ & $0.12655(16)$ & $0.10077(8)$ & $0.0276(3)$ \\
\hline H10 & $0.5629(17)$ & $0.178(2)$ & $0.0567(10)$ & $0.041(5)^{*}$ \\
\hline C11 & $0.51380(14)$ & $-0.00811(16)$ & $0.10078(8)$ & 0.0295 \\
\hline H11 & $0.4913(18)$ & $-0.0529(19)$ & $0.0555(10)$ & $0.040(5)^{*}$ \\
\hline $\mathrm{C} 12$ & $0.50215(15)$ & $-0.07840(15)$ & $0.16501(8)$ & $0.0312(3)$ \\
\hline H12 & $0.4716(17)$ & $-0.170(2)$ & $0.1652(10)$ & $0.037(5)^{*}$ \\
\hline $\mathrm{C} 13$ & $0.53114(14)$ & $-0.01448(15)$ & $0.22967(8)$ & 0.0279 \\
\hline H13 & $0.5240(17)$ & $-0.0659(18)$ & $0.2763(10)$ & $0.036(5)^{*}$ \\
\hline $\mathrm{C} 14$ & $0.72087(12)$ & $0.15479(13)$ & $0.44955(7)$ & 0.0220 \\
\hline $\mathrm{C} 15$ & $0.84103(15)$ & $0.10914(17)$ & $0.41681(10)$ & $0.0351(3)$ \\
\hline $\mathrm{H} 15 \mathrm{~A}$ & $0.907(2)$ & $0.149(2)$ & $0.4470(12)$ & $0.056(6)^{*}$ \\
\hline
\end{tabular}




$\begin{array}{lllll}\text { H15B } & 0.846(2) & 0.008(2) & 0.4173(12) & 0.058(6)^{*} \\ \text { H14C } & 0.8516(19) & 0.147(2) & 0.3658(12) & 0.051(6)^{*} \\ \text { C16 } & 0.71490(14) & 0.31393(14) & 0.58331(7) & 0.0282(3) \\ \text { H16A } & 0.723453 & 0.384753 & 0.620421 & 0.042^{*} \\ \text { H16B } & 0.791792 & 0.260036 & 0.580824 & 0.042^{*} \\ \text { H16C } & 0.645041 & 0.253596 & 0.595442 & 0.042^{*} \\ \text { C17 } & 0.60967(13) & 0.59215(14) & 0.32293(7) & 0.0245(3) \\ \text { C18 } & 0.63109(13) & 0.80140(14) & 0.54760(7) & 0.0231(3) \\ \text { H18A } & 0.6100(17) & 0.717(2) & 0.5740(10) & 0.037(5)^{*} \\ \text { H18B } & 0.5672(16) & 0.8694(18) & 0.5554(9) & 0.027(4)^{*} \\ \text { C19 } & 0.75457(13) & 0.85366(14) & 0.57467(7) & 0.0244(3) \\ \text { C20 } & 0.84825(15) & 0.98717(19) & 0.66682(9) & 0.0380(4) \\ \text { H20A } & 0.904(2) & 0.910(2) & 0.6807(12) & 0.053(6)^{*} \\ \text { H20B } & 0.896(2) & 1.047(2) & 0.6298(12) & 0.056(6)^{*} \\ \text { C21 } & 0.80775(18) & 1.0701(2) & 0.73071(10) & 0.0410(4) \\ \text { H21A } & 0.883(2) & 1.109(2) & 0.7550(13) & 0.065(7)^{*} \\ \text { H21B } & 0.762(2) & 1.014(2) & 0.7639(12) & 0.051(6)^{*} \\ \text { H21C } & 0.751(2) & 1.144(2) & 0.7167(11) & 0.049(6)^{*} \\ \end{array}$

Atomic displacement parameters $\left(\AA^{2}\right)$

\begin{tabular}{lllllll}
\hline & $U^{11}$ & $U^{22}$ & $U^{33}$ & $U^{12}$ & $U^{13}$ & $U^{23}$ \\
\hline S1 & $0.03027(19)$ & $0.01732(17)$ & $0.02199(18)$ & $0.00121(12)$ & $-0.00303(12)$ & $-0.00091(11)$ \\
O1 & $0.0316(5)$ & $0.0202(5)$ & $0.0367(6)$ & $0.0000(4)$ & $0.0043(4)$ & $0.0046(4)$ \\
O2 & $0.0248(5)$ & $0.0464(7)$ & $0.0389(6)$ & $0.0060(4)$ & $-0.0025(4)$ & $-0.0121(5)$ \\
O3 & $0.0289(5)$ & $0.0356(6)$ & $0.0266(5)$ & $0.0020(4)$ & $-0.0059(4)$ & $-0.0092(4)$ \\
N1 & $0.0257(6)$ & $0.0202(5)$ & $0.0208(6)$ & $-0.0021(4)$ & $-0.0033(4)$ & $-0.0004(4)$ \\
N2 & $0.0473(8)$ & $0.0295(6)$ & $0.0285(7)$ & $-0.0007(6)$ & $-0.0097(6)$ & $0.0023(5)$ \\
C1 & $0.0199(6)$ & $0.0200(6)$ & $0.0209(6)$ & $-0.0030(5)$ & $-0.0009(5)$ & $-0.0009(5)$ \\
C2 & $0.0203(6)$ & $0.0180(6)$ & $0.0224(6)$ & $-0.0021(5)$ & $-0.0018(5)$ & $0.0007(5)$ \\
C3 & $0.0224(6)$ & $0.0201(6)$ & $0.0220(7)$ & $-0.0025(5)$ & $-0.0032(5)$ & $0.0008(5)$ \\
C4 & $0.0199(6)$ & $0.0192(6)$ & $0.0211(6)$ & $-0.0021(5)$ & $-0.0023(5)$ & $-0.0002(5)$ \\
C5 & $0.0223(6)$ & $0.0203(6)$ & $0.0199(6)$ & $-0.0022(5)$ & $-0.0033(5)$ & $0.0012(5)$ \\
C6 & $0.0283(7)$ & $0.0222(6)$ & $0.0195(6)$ & $-0.0010(5)$ & $-0.0012(5)$ & $-0.0010(5)$ \\
C7 & $0.0281(7)$ & $0.0245(7)$ & $0.0193(6)$ & $-0.0024(5)$ & $0.0000(5)$ & $-0.0010(5)$ \\
C8 & $0.0233(6)$ & $0.0230(6)$ & $0.0201(6)$ & $-0.0001(5)$ & $-0.0010(5)$ & $-0.0028(5)$ \\
C9 & $0.0271(7)$ & $0.0233(7)$ & $0.0232(7)$ & $-0.0021(5)$ & $-0.0012(5)$ & $0.0003(5)$ \\
C10 & $0.0290(7)$ & $0.0329(7)$ & $0.0209(7)$ & $0.0010(6)$ & $-0.0022(5)$ & $0.0007(6)$ \\
C11 & $0.0337(7)$ & $0.0323(7)$ & $0.0226(7)$ & $0.0009(6)$ & $-0.0057(6)$ & $-0.0064(6)$ \\
C12 & $0.0418(8)$ & $0.0230(7)$ & $0.0287(8)$ & $-0.0048(6)$ & $-0.0045(6)$ & $-0.0046(6)$ \\
C13 & $0.0368(8)$ & $0.0240(7)$ & $0.0228(7)$ & $-0.0035(6)$ & $-0.0018(6)$ & $-0.0006(5)$ \\
C14 & $0.0244(6)$ & $0.0202(6)$ & $0.0214(6)$ & $-0.0002(5)$ & $-0.0042(5)$ & $-0.0005(5)$ \\
C15 & $0.0311(8)$ & $0.0294(8)$ & $0.0448(10)$ & $0.0052(6)$ & $0.0068(7)$ & $0.0029(7)$ \\
C16 & $0.0393(8)$ & $0.0239(7)$ & $0.0214(7)$ & $-0.0016(6)$ & $-0.0065(6)$ & $0.0022(5)$ \\
C17 & $0.0293(7)$ & $0.0211(6)$ & $0.0231(7)$ & $-0.0022(5)$ & $-0.0044(5)$ & $-0.0016(5)$ \\
C18 & $0.0257(7)$ & $0.0207(6)$ & $0.0230(7)$ & $0.0004(5)$ & $0.0007(5)$ & $-0.0027(5)$ \\
C19 & $0.0277(7)$ & $0.0223(6)$ & $0.0231(6)$ & $0.0038(5)$ & $-0.0038(5)$ & $-0.0008(5)$ \\
C20 & $0.0329(8)$ & $0.0429(9)$ & $0.0383(9)$ & $0.0026(7)$ & $-0.0140(7)$ & $-0.0119(7)$ \\
& & & & &
\end{tabular}




$\begin{array}{lllllll}\mathrm{C} 21 & 0.0458(10) & 0.0446(10) & 0.0324(9) & -0.0011(8) & -0.0100(7) & -0.0095(7)\end{array}$

Geometric parameters $\left(\AA,{ }^{\circ}\right)$

\begin{tabular}{|c|c|c|c|}
\hline $\mathrm{S} 1-\mathrm{C} 4$ & $1.7581(13)$ & C9-H9 & $0.960(19)$ \\
\hline $\mathrm{S} 1-\mathrm{C} 18$ & $1.7918(14)$ & $\mathrm{C} 10-\mathrm{C} 11$ & $1.389(2)$ \\
\hline $\mathrm{O} 1-\mathrm{C} 14$ & $1.2112(16)$ & $\mathrm{C} 10-\mathrm{H} 10$ & $0.96(2)$ \\
\hline $\mathrm{O} 2-\mathrm{C} 19$ & $1.2026(17)$ & $\mathrm{C} 11-\mathrm{C} 12$ & $1.381(2)$ \\
\hline $\mathrm{O} 3-\mathrm{C} 19$ & $1.3417(17)$ & $\mathrm{C} 11-\mathrm{H} 11$ & $0.98(2)$ \\
\hline $\mathrm{O} 3-\mathrm{C} 20$ & $1.4578(18)$ & $\mathrm{C} 12-\mathrm{C} 13$ & $1.388(2)$ \\
\hline $\mathrm{N} 1-\mathrm{C} 4$ & $1.3301(17)$ & $\mathrm{C} 12-\mathrm{H} 12$ & $0.956(19)$ \\
\hline $\mathrm{N} 1-\mathrm{C} 3$ & $1.3482(17)$ & $\mathrm{C} 13-\mathrm{H} 13$ & $1.003(19)$ \\
\hline $\mathrm{N} 2-\mathrm{C} 17$ & $1.1473(19)$ & $\mathrm{C} 14-\mathrm{C} 15$ & $1.4946(19)$ \\
\hline $\mathrm{C} 1-\mathrm{C} 2$ & $1.4025(18)$ & $\mathrm{C} 15-\mathrm{H} 15 \mathrm{~A}$ & $0.98(2)$ \\
\hline $\mathrm{C} 1-\mathrm{C} 5$ & $1.4038(18)$ & C15-H15B & $0.99(2)$ \\
\hline $\mathrm{C} 1-\mathrm{C} 6$ & $1.4723(18)$ & $\mathrm{C} 15-\mathrm{H} 14 \mathrm{C}$ & $1.02(2)$ \\
\hline $\mathrm{C} 2-\mathrm{C} 3$ & $1.4032(18)$ & C16-H16A & 0.9800 \\
\hline $\mathrm{C} 2-\mathrm{C} 14$ & $1.5130(17)$ & C16-H16B & 0.9800 \\
\hline $\mathrm{C} 3-\mathrm{C} 16$ & $1.5013(18)$ & $\mathrm{C} 16-\mathrm{H} 16 \mathrm{C}$ & 0.9800 \\
\hline $\mathrm{C} 4-\mathrm{C} 5$ & $1.4032(18)$ & $\mathrm{C} 18-\mathrm{C} 19$ & $1.5061(19)$ \\
\hline $\mathrm{C} 5-\mathrm{C} 17$ & $1.4378(18)$ & C18-H18A & $0.988(19)$ \\
\hline $\mathrm{C} 6-\mathrm{C} 7$ & $1.332(2)$ & C18-H18B & $0.965(17)$ \\
\hline C6- 6 6 & $0.968(19)$ & $\mathrm{C} 20-\mathrm{C} 21$ & $1.500(2)$ \\
\hline $\mathrm{C} 7-\mathrm{C} 8$ & $1.4669(18)$ & $\mathrm{C} 20-\mathrm{H} 20 \mathrm{~A}$ & $1.00(2)$ \\
\hline $\mathrm{C} 7-\mathrm{H} 7$ & $0.961(18)$ & $\mathrm{C} 20-\mathrm{H} 20 \mathrm{~B}$ & $1.04(2)$ \\
\hline $\mathrm{C} 8-\mathrm{C} 13$ & $1.3958(19)$ & $\mathrm{C} 21-\mathrm{H} 21 \mathrm{~A}$ & $1.00(2)$ \\
\hline $\mathrm{C} 8-\mathrm{C} 9$ & $1.4016(19)$ & $\mathrm{C} 21-\mathrm{H} 21 \mathrm{~B}$ & $0.96(2)$ \\
\hline $\mathrm{C} 9-\mathrm{C} 10$ & $1.385(2)$ & $\mathrm{C} 21-\mathrm{H} 21 \mathrm{C}$ & $0.97(2)$ \\
\hline $\mathrm{C} 4-\mathrm{S} 1-\mathrm{C} 18$ & $102.24(6)$ & $\mathrm{C} 12-\mathrm{C} 13-\mathrm{H} 13$ & $120.2(10)$ \\
\hline $\mathrm{C} 19-\mathrm{O} 3-\mathrm{C} 20$ & $115.85(11)$ & $\mathrm{C} 8-\mathrm{C} 13-\mathrm{H} 13$ & $119.0(10)$ \\
\hline $\mathrm{C} 4-\mathrm{N} 1-\mathrm{C} 3$ & $118.68(11)$ & $\mathrm{O} 1-\mathrm{C} 14-\mathrm{C} 15$ & $122.21(12)$ \\
\hline $\mathrm{C} 2-\mathrm{C} 1-\mathrm{C} 5$ & $116.93(11)$ & $\mathrm{O} 1-\mathrm{C} 14-\mathrm{C} 2$ & $119.95(12)$ \\
\hline $\mathrm{C} 2-\mathrm{C} 1-\mathrm{C} 6$ & $124.86(12)$ & $\mathrm{C} 15-\mathrm{C} 14-\mathrm{C} 2$ & $117.77(12)$ \\
\hline $\mathrm{C} 5-\mathrm{C} 1-\mathrm{C} 6$ & $118.14(11)$ & $\mathrm{C} 14-\mathrm{C} 15-\mathrm{H} 15 \mathrm{~A}$ & $105.7(13)$ \\
\hline $\mathrm{C} 1-\mathrm{C} 2-\mathrm{C} 3$ & $119.21(12)$ & $\mathrm{C} 14-\mathrm{C} 15-\mathrm{H} 15 \mathrm{~B}$ & $109.9(13)$ \\
\hline $\mathrm{C} 1-\mathrm{C} 2-\mathrm{C} 14$ & $122.32(11)$ & $\mathrm{H} 15 \mathrm{~A}-\mathrm{C} 15-\mathrm{H} 15 \mathrm{~B}$ & $110.5(18)$ \\
\hline $\mathrm{C} 3-\mathrm{C} 2-\mathrm{C} 14$ & $118.47(11)$ & $\mathrm{C} 14-\mathrm{C} 15-\mathrm{H} 14 \mathrm{C}$ & $111.5(12)$ \\
\hline $\mathrm{N} 1-\mathrm{C} 3-\mathrm{C} 2$ & $122.74(12)$ & $\mathrm{H} 15 \mathrm{~A}-\mathrm{C} 15-\mathrm{H} 14 \mathrm{C}$ & $107.7(17)$ \\
\hline $\mathrm{N} 1-\mathrm{C} 3-\mathrm{C} 16$ & $114.90(11)$ & $\mathrm{H} 15 \mathrm{~B}-\mathrm{C} 15-\mathrm{H} 14 \mathrm{C}$ & $111.2(17)$ \\
\hline $\mathrm{C} 2-\mathrm{C} 3-\mathrm{C} 16$ & $122.34(12)$ & $\mathrm{C} 3-\mathrm{C} 16-\mathrm{H} 16 \mathrm{~A}$ & 109.5 \\
\hline $\mathrm{N} 1-\mathrm{C} 4-\mathrm{C} 5$ & $122.14(12)$ & $\mathrm{C} 3-\mathrm{C} 16-\mathrm{H} 16 \mathrm{~B}$ & 109.5 \\
\hline $\mathrm{N} 1-\mathrm{C} 4-\mathrm{S} 1$ & $120.40(10)$ & $\mathrm{H} 16 \mathrm{~A}-\mathrm{C} 16-\mathrm{H} 16 \mathrm{~B}$ & 109.5 \\
\hline $\mathrm{C} 5-\mathrm{C} 4-\mathrm{S} 1$ & $117.46(10)$ & $\mathrm{C} 3-\mathrm{C} 16-\mathrm{H} 16 \mathrm{C}$ & 109.5 \\
\hline $\mathrm{C} 4-\mathrm{C} 5-\mathrm{C} 1$ & $120.22(12)$ & $\mathrm{H} 16 \mathrm{~A}-\mathrm{C} 16-\mathrm{H} 16 \mathrm{C}$ & 109.5 \\
\hline $\mathrm{C} 4-\mathrm{C} 5-\mathrm{C} 17$ & $119.59(12)$ & $\mathrm{H} 16 \mathrm{~B}-\mathrm{C} 16-\mathrm{H} 16 \mathrm{C}$ & 109.5 \\
\hline $\mathrm{C} 1-\mathrm{C} 5-\mathrm{C} 17$ & $120.16(12)$ & $\mathrm{N} 2-\mathrm{C} 17-\mathrm{C} 5$ & $178.94(16)$ \\
\hline $\mathrm{C} 7-\mathrm{C} 6-\mathrm{C} 1$ & $124.98(12)$ & $\mathrm{C} 19-\mathrm{C} 18-\mathrm{S} 1$ & $113.86(10)$ \\
\hline
\end{tabular}




\begin{tabular}{|c|c|c|c|}
\hline $\mathrm{C} 7-\mathrm{C} 6-\mathrm{H} 6$ & $120.3(11)$ & $\mathrm{C} 19-\mathrm{C} 18-\mathrm{H} 18 \mathrm{~A}$ & $108.7(11)$ \\
\hline $\mathrm{C} 1-\mathrm{C} 6-\mathrm{H} 6$ & $114.6(11)$ & $\mathrm{S} 1-\mathrm{C} 18-\mathrm{H} 18 \mathrm{~A}$ & $107.8(11)$ \\
\hline $\mathrm{C} 6-\mathrm{C} 7-\mathrm{C} 8$ & $126.26(13)$ & $\mathrm{C} 19-\mathrm{C} 18-\mathrm{H} 18 \mathrm{~B}$ & $110.0(10)$ \\
\hline $\mathrm{C} 6-\mathrm{C} 7-\mathrm{H} 7$ & $118.6(11)$ & $\mathrm{S} 1-\mathrm{C} 18-\mathrm{H} 18 \mathrm{~B}$ & $106.7(10)$ \\
\hline $\mathrm{C} 8-\mathrm{C} 7-\mathrm{H} 7$ & $115.1(11)$ & $\mathrm{H} 18 \mathrm{~A}-\mathrm{C} 18-\mathrm{H} 18 \mathrm{~B}$ & $109.7(14)$ \\
\hline $\mathrm{C} 13-\mathrm{C} 8-\mathrm{C} 9$ & $118.47(12)$ & $\mathrm{O} 2-\mathrm{C} 19-\mathrm{O} 3$ & $124.17(13)$ \\
\hline $\mathrm{C} 13-\mathrm{C} 8-\mathrm{C} 7$ & $118.34(12)$ & $\mathrm{O} 2-\mathrm{C} 19-\mathrm{C} 18$ & $126.38(13)$ \\
\hline $\mathrm{C} 9-\mathrm{C} 8-\mathrm{C} 7$ & $123.18(12)$ & $\mathrm{O} 3-\mathrm{C} 19-\mathrm{C} 18$ & $109.43(11)$ \\
\hline $\mathrm{C} 10-\mathrm{C} 9-\mathrm{C} 8$ & $120.64(13)$ & $\mathrm{O} 3-\mathrm{C} 20-\mathrm{C} 21$ & $107.39(14)$ \\
\hline $\mathrm{C} 10-\mathrm{C} 9-\mathrm{H} 9$ & $119.7(11)$ & $\mathrm{O} 3-\mathrm{C} 20-\mathrm{H} 20 \mathrm{~A}$ & $108.7(13)$ \\
\hline $\mathrm{C} 8-\mathrm{C} 9-\mathrm{H} 9$ & $119.7(11)$ & $\mathrm{C} 21-\mathrm{C} 20-\mathrm{H} 20 \mathrm{~A}$ & $112.2(13)$ \\
\hline $\mathrm{C} 9-\mathrm{C} 10-\mathrm{C} 11$ & $120.03(13)$ & $\mathrm{O} 3-\mathrm{C} 20-\mathrm{H} 20 \mathrm{~B}$ & $110.0(12)$ \\
\hline $\mathrm{C} 9-\mathrm{C} 10-\mathrm{H} 10$ & $118.6(12)$ & $\mathrm{C} 21-\mathrm{C} 20-\mathrm{H} 20 \mathrm{~B}$ & $111.1(12)$ \\
\hline $\mathrm{C} 11-\mathrm{C} 10-\mathrm{H} 10$ & $121.4(12)$ & $\mathrm{H} 20 \mathrm{~A}-\mathrm{C} 20-\mathrm{H} 20 \mathrm{~B}$ & $107.4(17)$ \\
\hline $\mathrm{C} 12-\mathrm{C} 11-\mathrm{C} 10$ & $119.98(13)$ & $\mathrm{C} 20-\mathrm{C} 21-\mathrm{H} 21 \mathrm{~A}$ & $109.1(13)$ \\
\hline $\mathrm{C} 12-\mathrm{C} 11-\mathrm{H} 11$ & $119.8(11)$ & $\mathrm{C} 20-\mathrm{C} 21-\mathrm{H} 21 \mathrm{~B}$ & $110.5(13)$ \\
\hline $\mathrm{C} 10-\mathrm{C} 11-\mathrm{H} 11$ & $120.2(11)$ & $\mathrm{H} 21 \mathrm{~A}-\mathrm{C} 21-\mathrm{H} 21 \mathrm{~B}$ & $109.7(18)$ \\
\hline $\mathrm{C} 11-\mathrm{C} 12-\mathrm{C} 13$ & $120.18(13)$ & $\mathrm{C} 20-\mathrm{C} 21-\mathrm{H} 21 \mathrm{C}$ & $111.6(12)$ \\
\hline $\mathrm{C} 11-\mathrm{C} 12-\mathrm{H} 12$ & $120.1(11)$ & $\mathrm{H} 21 \mathrm{~A}-\mathrm{C} 21-\mathrm{H} 21 \mathrm{C}$ & $110.1(18)$ \\
\hline $\mathrm{C} 13-\mathrm{C} 12-\mathrm{H} 12$ & $119.7(11)$ & $\mathrm{H} 21 \mathrm{~B}-\mathrm{C} 21-\mathrm{H} 21 \mathrm{C}$ & $105.8(18)$ \\
\hline $\mathrm{C} 12-\mathrm{C} 13-\mathrm{C} 8$ & $120.69(13)$ & & \\
\hline $\mathrm{C} 5-\mathrm{C} 1-\mathrm{C} 2-\mathrm{C} 3$ & $2.15(18)$ & $\mathrm{C} 5-\mathrm{C} 1-\mathrm{C} 6-\mathrm{C} 7$ & $-140.67(14)$ \\
\hline $\mathrm{C} 6-\mathrm{C} 1-\mathrm{C} 2-\mathrm{C} 3$ & $-175.01(12)$ & $\mathrm{C} 1-\mathrm{C} 6-\mathrm{C} 7-\mathrm{C} 8$ & $173.53(13)$ \\
\hline $\mathrm{C} 5-\mathrm{C} 1-\mathrm{C} 2-\mathrm{C} 14$ & $-176.70(11)$ & $\mathrm{C} 6-\mathrm{C} 7-\mathrm{C} 8-\mathrm{C} 13$ & $172.91(14)$ \\
\hline $\mathrm{C} 6-\mathrm{C} 1-\mathrm{C} 2-\mathrm{C} 14$ & $6.15(19)$ & $\mathrm{C} 6-\mathrm{C} 7-\mathrm{C} 8-\mathrm{C} 9$ & $-8.2(2)$ \\
\hline $\mathrm{C} 4-\mathrm{N} 1-\mathrm{C} 3-\mathrm{C} 2$ & $1.39(19)$ & $\mathrm{C} 13-\mathrm{C} 8-\mathrm{C} 9-\mathrm{C} 10$ & $0.5(2)$ \\
\hline $\mathrm{C} 4-\mathrm{N} 1-\mathrm{C} 3-\mathrm{C} 16$ & $-179.73(12)$ & $\mathrm{C} 7-\mathrm{C} 8-\mathrm{C} 9-\mathrm{C} 10$ & $-178.32(13)$ \\
\hline $\mathrm{C} 1-\mathrm{C} 2-\mathrm{C} 3-\mathrm{N} 1$ & $-3.20(19)$ & $\mathrm{C} 8-\mathrm{C} 9-\mathrm{C} 10-\mathrm{C} 11$ & $0.0(2)$ \\
\hline $\mathrm{C} 14-\mathrm{C} 2-\mathrm{C} 3-\mathrm{N} 1$ & $175.69(12)$ & $\mathrm{C} 9-\mathrm{C} 10-\mathrm{C} 11-\mathrm{C} 12$ & $-0.6(2)$ \\
\hline $\mathrm{C} 1-\mathrm{C} 2-\mathrm{C} 3-\mathrm{C} 16$ & $177.99(12)$ & $\mathrm{C} 10-\mathrm{C} 11-\mathrm{C} 12-\mathrm{C} 13$ & $0.6(2)$ \\
\hline $\mathrm{C} 14-\mathrm{C} 2-\mathrm{C} 3-\mathrm{C} 16$ & $-3.12(19)$ & $\mathrm{C} 11-\mathrm{C} 12-\mathrm{C} 13-\mathrm{C} 8$ & $0.0(2)$ \\
\hline $\mathrm{C} 3-\mathrm{N} 1-\mathrm{C} 4-\mathrm{C} 5$ & $1.39(19)$ & $\mathrm{C} 9-\mathrm{C} 8-\mathrm{C} 13-\mathrm{C} 12$ & $-0.5(2)$ \\
\hline $\mathrm{C} 3-\mathrm{N} 1-\mathrm{C} 4-\mathrm{S} 1$ & $-178.47(10)$ & $\mathrm{C} 7-\mathrm{C} 8-\mathrm{C} 13-\mathrm{C} 12$ & $178.38(14)$ \\
\hline $\mathrm{C} 18-\mathrm{S} 1-\mathrm{C} 4-\mathrm{N} 1$ & $-5.66(12)$ & $\mathrm{C} 1-\mathrm{C} 2-\mathrm{C} 14-\mathrm{O} 1$ & $-114.75(15)$ \\
\hline $\mathrm{C} 18-\mathrm{S} 1-\mathrm{C} 4-\mathrm{C} 5$ & $174.48(10)$ & $\mathrm{C} 3-\mathrm{C} 2-\mathrm{C} 14-\mathrm{O} 1$ & $66.40(17)$ \\
\hline $\mathrm{N} 1-\mathrm{C} 4-\mathrm{C} 5-\mathrm{C} 1$ & $-2.31(19)$ & $\mathrm{C} 1-\mathrm{C} 2-\mathrm{C} 14-\mathrm{C} 15$ & $68.12(17)$ \\
\hline $\mathrm{S} 1-\mathrm{C} 4-\mathrm{C} 5-\mathrm{C} 1$ & $177.55(10)$ & $\mathrm{C} 3-\mathrm{C} 2-\mathrm{C} 14-\mathrm{C} 15$ & $-110.73(15)$ \\
\hline $\mathrm{N} 1-\mathrm{C} 4-\mathrm{C} 5-\mathrm{C} 17$ & $175.55(12)$ & $\mathrm{C} 4-\mathrm{S} 1-\mathrm{C} 18-\mathrm{C} 19$ & $98.96(10)$ \\
\hline $\mathrm{S} 1-\mathrm{C} 4-\mathrm{C} 5-\mathrm{C} 17$ & $-4.59(17)$ & $\mathrm{C} 20-\mathrm{O} 3-\mathrm{C} 19-\mathrm{O} 2$ & $0.6(2)$ \\
\hline $\mathrm{C} 2-\mathrm{C} 1-\mathrm{C} 5-\mathrm{C} 4$ & $0.44(18)$ & $\mathrm{C} 20-\mathrm{O} 3-\mathrm{C} 19-\mathrm{C} 18$ & $179.32(12)$ \\
\hline $\mathrm{C} 6-\mathrm{C} 1-\mathrm{C} 5-\mathrm{C} 4$ & $177.79(12)$ & $\mathrm{S} 1-\mathrm{C} 18-\mathrm{C} 19-\mathrm{O} 2$ & $-26.34(19)$ \\
\hline $\mathrm{C} 2-\mathrm{C} 1-\mathrm{C} 5-\mathrm{C} 17$ & $-177.42(12)$ & $\mathrm{S} 1-\mathrm{C} 18-\mathrm{C} 19-\mathrm{O} 3$ & $154.93(10)$ \\
\hline $\mathrm{C} 6-\mathrm{C} 1-\mathrm{C} 5-\mathrm{C} 17$ & $-0.06(18)$ & $\mathrm{C} 19-\mathrm{O} 3-\mathrm{C} 20-\mathrm{C} 21$ & $179.23(14)$ \\
\hline $\mathrm{C} 2-\mathrm{C} 1-\mathrm{C} 6-\mathrm{C} 7$ & $36.5(2)$ & & \\
\hline
\end{tabular}


supporting information

Hydrogen-bond geometry $\left(\AA,{ }^{\circ}\right)$

\begin{tabular}{lllll}
\hline$D-\mathrm{H} \cdots A$ & $D-\mathrm{H}$ & $\mathrm{H} \cdots A$ & $D \cdots A$ & $D-\mathrm{H} \cdots A$ \\
\hline $\mathrm{C} 15-\mathrm{H} 15 A \cdots \mathrm{O} 2^{\mathrm{i}}$ & $0.98(2)$ & $2.56(2)$ & $3.375(2)$ & $139.9(17)$ \\
$\mathrm{C} 18-\mathrm{H} 18 B \cdots \mathrm{O} 1^{\mathrm{ii}}$ & $0.965(17)$ & $2.493(17)$ & $3.2989(17)$ & $140.9(13)$ \\
\hline
\end{tabular}

Symmetry codes: (i) $-x+2,-y+1,-z+1$; (ii) $-x+1,-y+1,-z+1$. 